\title{
Tumor Estromal Gastrointestinal: Experiência no Tratamento da Doença Localizada e Avançada no Hospital de Clínicas da Universidade Federal do Paraná Gastrointestinal Stromal Tumor: Experience in the Treatment of Localized and Advanced Disease at the Clinical Hospital of Parana Federal University Tumor del Estroma Gastrointestinal: Experiencia en el Tratamiento de la Enfermedad Localizada y Avanzada en el Hospital de Clínicas de la Universidad Federal de Paraná
}

\author{
Karina Costa Maia Vianna', Sérgio Lunardon Padilha², Bianca Ramos Ferronatto ${ }^{3}$, Lucas Fernando Uratani ${ }^{3}$, Felipe Augusto Santiago de Almeida ${ }^{3}$, \\ José Zanis Neto ${ }^{4}$
}

\section{Resumo}

Introduçáo: Tumores estromais gastrointestinais (GIST) são neoplasias raras que se originam das células intersticiais de Cajal. Objetivo: Descrever a experiência do Hospital de Clínicas de Curitiba no tratamento do GIST localizado e avançado, com análise das características clínicas e anatomopatológicas e uso do imatinibe. Método: Estudo retrospectivo com 32 pacientes com diagnóstico por imuno-histoquímica, c-Kit positivo, no período de 2003 a 2008 . Resultados: Idade mediana: 66 anos; tamanho mediano do tumor de $8,4 \mathrm{~cm}$; e as localizaçóes mais frequentes foram estômago em 46,9\% e intestino delgado em 40,9\%. Pacientes com alto risco de agressividade: $37,5 \%$; apresentavam doença localizada no diagnóstico 23 pacientes: 39,1\% recaíram e 9 com doença avançada. O seguimento mediano foi de 43,7 meses, com sobrevida global em 5 anos no grupo total de 56,2\%. Na doença localizada, a sobrevida global em 5 anos foi de $73,8 \%$ e na avançada de $37,5 \%(p=0,03)$. Não ocorreu impacto dos fatores prognósticos na sobrevida. Utilizou-se o mesilato de imatinibe em 16 pacientes: 43,8\% por metástase inicial, 37,5\% recaída a distância, 12,5\% recaída local e $6,2 \%$ margem comprometida. A sobrevida global com uso do imatinibe mediana foi de 53 meses e a sobrevida livre de primeira progressão de 32,9 meses. Houve boa tolerabilidade ao imatinibe e apenas dois pacientes utilizaram o sunitinibe. Conclusáo: A maioria dos tumores era grande, de localização gástrica e de alto risco de agressividade. A taxa de recaída na doença localizada foi alta. E a sobrevida global dos pacientes de doença localizada e que utilizaram o imatinibe foi considerada satisfatória.

Palavras-chave: Tumores do Estroma Gastrointestinal; Antineoplásicos/uso terapêutico; Mesilatos; Proteínas ProtoOncogênicas c-kit; Imunoistoquímica; Estudos Retrospectivos

Estudo extraído de dissertação de mestrado em Medicina Interna e Ciências da Saúde Hospital de Clínicas da Universidade Federal do Paraná (UFPR). Serviço de Hematologia e Oncologia.

${ }^{1}$ Oncologista Clínica do Serviço de Hematologia e Oncologia do Hospital de Clínicas da UFPR. Mestre em Medicina Interna e Ciências da Saúde no Hospital de Clínicas da UFPR. Curitiba (PR), Brasil.

${ }^{2}$ Mestre em Medicina Interna e Ciências da Saúde no Hospital de Clínicas da UFPR e Professor assistente da Disciplina de Hematologia e Oncologia da UFPR. Curitiba (PR), Brasil.

${ }^{3}$ Acadêmicos do curso de Graduaçáo em Medicina da UFPR. Curitiba (PR), Brasil.

${ }^{4}$ Doutor em Medicina Interna e Ciências da Saúde no Hospital de Clínicas da UFPR. Professor Associado da Disciplina de Hematologia e Oncologia da UFPR. Chefe do Serviço de Transplante de Medula Óssea do Hospital de Clínicas. Curitiba (PR), Brasil.

Endereço para correspondência: Karina Costa Maia Vianna. Ambulatório de Hematologia e Oncologia (SAM 16). Hospital de Clínicas da UFPR. Avenida General Carneiro, 181. Curitiba (PR), Brasil. CEP: 80060-900.E-mail:kcmvianna@yahoo.com.br 


\section{INTRODUÇÃO}

Os tumores estromais gastrointestinais (GIST) originam-se das células intersticiais de Cajal e consistem na neoplasia mesenquimal mais comum do trato gastrointestinal, $5 \%$ a $6 \%$ de todos os sarcomas e $1 \%$ a $3 \%$ de todas as neoplasias do trato digestivo ${ }^{1-3}$.

A estimativa nos Estados Unidos é de 4.000 a 6.000 novos casos/ano e uma incidência de 6,8 casos por 1 milhão; e 11 a 14,5 casos em cada 1 milhão de pessoas na Europa, não tendo sido identificados fatores de risco para sua ocorrência ou associação com outras neoplasias ${ }^{1,4-5}$. Não existe uma estimativa do GIST no Brasil. A maioria desses tumores apresenta mutação do gene Kit, de $85 \%$ a $88 \%$ dos casos, com mais frequência no éxon 11 ; o restante, mutaçáo no receptor alfa do fator de crescimento derivado das plaquetas (PDGFR alfa), de 5\% a 7\%, e ausência de mutaçóes detectadas em $10 \%$ dos tumores ${ }^{1,6-7}$.

O manejo da doença localizada consiste na ressecção cirúrgica completa, sendo a única modalidade com capacidade de cura. Apesar disso, mais de 50\% dos pacientes submetidosà cirurgia irão recidivar, principalmente no peritônio e fígado ${ }^{8-9}$. $\mathrm{Na}$ doença metastática, o tratamento realizado no passado em tumores isolados era a cirurgia, com uma alta taxa de recaída, uma vez que esses tumores respondiam pobremente à quimioterapia sistêmica ou radioterapia ${ }^{1-2,9}$.

A descoberta do mesilato de imatinibe (Glivec ${ }^{\odot}$ ) revolucionou o tratamento do câncer, por ter sido a primeira droga com alvo molecular. O conhecimento de mutaçóes do gene Kit na gênese e progressão do GIST proporcionou a utilização desse inibidor competitivo múltiplo de enzima tirosino-quinase nessa neoplasia ${ }^{1,7-8}$. O primeiro relato de atividade da droga nesse tumor foi publicado em $2001^{10}$. A sobrevida mediana na doença avançada que atingia por volta de 15 meses aumentou para 55 a 57 meses $^{7,11-12}$. Embora um grande ganho na qualidade de vida e sobrevida tenha ocorrido nesses pacientes, não existe a cura para a maioria dos pacientes na doença metastática. Esses pacientes irão desenvolver resistência, decorrendo normalmente de mutaçóes adquiridas ${ }^{1}$.

Diante do exposto, torna-se importante o conhecimento de como o GIST comporta-se em uma populaçáo atendida em um centro único brasileiro, comparando-se a dados recentemente conhecidos de centros americanos, europeus e asiáticos. Este estudo tem o objetivo de descrever a experiência do Hospital de Clínicas de Curitiba no tratamento do GIST localizado e avançado, com análise das características clínicas e anatomopatológicas e uso do imatinibe.

\section{MÉTODO}

Estudo observacional, transversal, descritivo e retrospectivo realizado no Hospital de Clínicas da Universidade Federal do Paraná, através da identificação de 32 pacientes com diagnóstico por imuno-histoquímica de GIST, no período de janeiro de 2003 a dezembro de 2008. No Serviço de Anatomia Patológica, foram identificados os pacientes positivos para a pesquisa da proteína c-Kit (CD117) por imuno-histoquímica e diagnóstico conclusivo de GIST. Foram acrescentados a essa amostra os pacientes com diagnóstico de GIST em serviços externos de patologia, com as lâminas revisadas no Serviço de Anatomia Patológica do Hospital de Clínicas, e início do acompanhamento no ambulatório de oncologia no período de 2003 a 2008. Os prontuários hospitalares e eletrônicos dos pacientes consistiram na fonte de dados.

Foram analisados dois grupos de pacientes, o primeiro de pacientes com doença localizada no diagnóstico inicial, sendo considerado com acometimento do órgáo primário ressecável, e o segundo com doença avançada, sendo considerados os pacientes com diagnóstico de metástase a distância (fígado, peritônio, pulmão e pleura) ou doença irressecável na apresentaçáo clínica inicial. Foram avaliadas variáveis clínicas e anatomopatológicas relacionadas ao GIST e a sobrevida global e sobrevida livre de doença. Uma das variáveis avaliadas foi a categorização de risco pelo National Institutes of Health (NIH), que consiste no risco de comportamento agressivo, sendo muito baixo risco tumores com tamanho menor do que $2 \mathrm{~cm}$ e índice mitótico menor do que 5 por 50 campos de grande aumento (CGA), baixo risco com tamanho de 2 a $5 \mathrm{~cm}$ e índice menor 5 por 50 CGA, risco intermediário com tamanho menor do que $5 \mathrm{~cm}$ e índice entre 6 e 10 e 5 a $10 \mathrm{~cm}$ e índice menor que 5 por $50 \mathrm{CGA}$, e alto risco com tamanho maior que $5 \mathrm{~cm}$ e índice maior que 5 por 50 CGA, qualquer tumor maior que $10 \mathrm{~cm}$ e qualquer índice maior que 10 por 50 CGA. Uma outra análise realizada foi dos pacientes que utilizaram o mesilato de imatinibe, com relação às indicaçôes, respostas e tolerância, além da sobrevida livre de progressão e global deste grupo.

A avaliação de resposta foi realizada pelos critérios de avaliaçấo de resposta dos tumores sólidos (RECIST): resposta completa, como desaparecimento radiológico da lesão; resposta parcial como redução de mais de 30\% do tamanho tumoral; doença estável como ausência de alteração ou redução de menos de $30 \%$ do tamanho tumoral e progressáo como aumento de mais de $20 \%$ no tamanho da lesão ou novas lesóes. $\mathrm{Na}$ sobrevida livre de doença, foram considerados o tempo entre o diagnóstico e a recaída ou óbito; na sobrevida livre de progressão, o tempo entre o início do imatinibe e a progressão ou óbito; e na sobrevida global, o tempo entre o diagnóstico e o óbito ou se vivo a data do censo.

A análise estatística foi realizada através do programa GraphPad Prism 5.0. A sobrevida foi estimada pelo método de Kaplan-Meier e as diferenças estatísticas 
entre os grupos foram analisadas pelo Teste de Log-rank (Mantel-Cox). O nível de significância estatística utilizada foi $\mathrm{p}<0,05$.

Este estudo foi avaliado e aprovado pelo Comitê de Ética em Pesquisa do Hospital de Clínicas da Universidade Federal do Paraná cadastrado sob o número 2185.079/2010-04.

\section{RESULTADOS}

\section{CARACTERISTICAS CLIINICAS E HISTOPATOLÓGICAS}

No período de janeiro de 2003 a dezembro de 2008, 32 pacientes foram diagnosticados e tratados com GIST (Tabela 1). A idade mediana foi de 59 anos, variando de 29 a 79 anos. Dos 32 pacientes, 16 eram homens e 16 eram mulheres. Os sintomas mais comuns na apresentação foram dor abdominal em 17 pacientes $(53,1 \%)$, seguido de sangramento gastrointestinal em sete pacientes $(21,8 \%)$, emagrecimento em cinco pacientes $(15,6 \%)$, astenia em três pacientes $(9,3 \%)$, massa abdominal palpável em dois pacientes $(6,2 \%)$ e obstipação em dois pacientes $(6,2 \%)$; do total, três pacientes eram assintomáticos $(9,3 \%)$. O sítio primário mais comum foi o estômago em 15 pacientes (46,9\%), seguido do intestino delgado em 13 pacientes $(40,6 \%)$, reto em dois pacientes $(6,3 \%)$ e esôfago em um paciente $(3,2 \%)$. Nos pacientes com doença localizada inicial, o sítio primário foi o estômago em $54,1 \%$ e intestino delgado em $39,1 \%$ e, nos pacientes com doença metastática inicial, 44,5\% eram primários do intestino delgado e 33,3\% primários do estômago.

A imuno-histoquímica foi positiva para CD117 em $100 \%$ e para CD34 em $81,2 \%$. O tamanho mediano dos tumores foi de $8,4 \mathrm{~cm}$, variando de 2 a $16 \mathrm{~cm}$, com uma mediana de $8 \mathrm{~cm}$ para a doença localizada e $9,8 \mathrm{~cm}$ para a doença avançada. O índice mitótico foi menor do que 5 por 50 campos de grande aumento em $58 \%$, maior do que 5 em 28,1\% e desconhecido em 21,9\%. $\mathrm{Na}$ categorização de risco pelo $\mathrm{NIH}$, nenhum caso foi avaliado como muito baixo risco, $15,6 \%$ como baixo risco, $18,7 \%$ como risco intermediário, $37,5 \%$ como alto risco, $18,7 \%$ não avaliável por metástase e $9,4 \%$ desconhecido. Apesar de cinco casos desconhecidos de índice mitótico na doença localizada, em dois, foi possível categorização de risco devido ao tamanho do tumor ser maior do que $5 \mathrm{~cm}$.

\section{TRATAMENTO E EVOLUÇÃO}

Dos 23 pacientes com doença localizada inicialmente, 22 foram submetidos à cirurgia de ressecção do tumor primário, quatro apresentavam margens cirúrgicas comprometidas no exame anatomopatológico $(18,1 \%)$ e um paciente faleceu por infecção de prótese ortopédica antes da cirurgia. Destes, um paciente recebeu imatinibe adjuvante devido às margens comprometidas. Ocorreram nove recaídas nesse grupo $(39,1 \%)$, seis recaídas em fígado, uma recaída em peritônio e duas recaídas locais, os três pacientes com margens comprometidas, que não utilizaram o imatinibe, recaíram. O tempo mediano para recaída foi de 10,8 meses.

Dos nove pacientes com doença avançada inicial, seis apresentavam metástase apenas em fígado, um em fígado e peritônio, um em fígado e pleura e um apenas em peritônio. Destes, sete pacientes receberam o imatinibe e dois não receberam por perda do seguimento.

\section{ANÁLISE DE SOBREVIDA}

O acompanhamento mediano foi de 43,7 meses, variando de 1,8 a 103,4 meses. No ponto utilizado como censo, do grupo total, 19 pacientes estavam vivos (59,3\%); no grupo da doença localizada, 17 pacientes estavam vivos $(73,9 \%)$; e no grupo da doença avançada, dois estavam vivos $(22,2 \%)$. Dos 12 pacientes que foram a óbito, a causa do óbito foi relacionada à progressão da doença em nove pacientes (75\%) e, em três pacientes, foram de outras causas, dois pacientes por septicemia e um por crise hipertensiva. Do grupo total, quatro pacientes perderam o seguimento ambulatorial (14,5\%), sabendo-se por contato telefônico que três destes encontravam-se vivos.

A sobrevida global em 5 anos foi de $56,2 \%$ e a sobrevida livre de progressão em 5 anos de 49,7\% (Figura 1). A sobrevida global em 5 anos da doença localizada inicial foi de $73,8 \%$ e a sobrevida livre de doença em 5 anos de 73,2\% (Figura 1). A sobrevida global em 5 anos da doença avançada inicial foi de $37,5 \%$, com mediana de 47,3 meses, e a sobrevida livre de progressáo em 4 anos de $28,5 \%$, com mediana de 32,9 meses. Na comparação da sobrevida global entre os pacientes com doença localizada e avançada, foi estatisticamente significante maior a sobrevida para a doença localizada $(\mathrm{p}=0,03)$ (Figura 1$)$.

Foram testados, na análise univariada, alguns fatores prognósticos (idade, sexo, tamanho tumoral, localização anatômica, grau de mitose, risco de agressividade, margem cirúrgica e CD34 positivo) do GIST localizado inicial, na possibilidade de impacto adverso estatisticamente significante na sobrevida livre de doença e acrescentado na avaliação da recaída na sobrevida global. Não houve diferença estatística em nenhum fator estudado (Tabela 2). Foi encontrada uma tendência $(\mathrm{p}=0,09)$ no grau de mitose e, quando retirados os dois pacientes com óbito precoce ( 0,33 meses), após o diagnóstico do GIST, o fator se tornou adverso $(\mathrm{p}=0,03)$ na sobrevida global.

\section{TERAPIA COM O IMATINIBE}

Dos 32 pacientes, 16 utilizaram o mesilato de imatinibe (50\%); sete pacientes por doença metastática 
Tabela 1. Características dos pacientes

\begin{tabular}{|c|c|c|c|c|c|c|}
\hline \multirow{2}{*}{$\begin{array}{l}\text { Características } \\
\text { dos pacientes }\end{array}$} & \multicolumn{2}{|c|}{$\begin{array}{c}\text { Total } \\
(n=32)\end{array}$} & \multicolumn{2}{|c|}{$\begin{array}{c}\text { GIST localizado } \\
(n=23)\end{array}$} & \multicolumn{2}{|c|}{$\begin{array}{c}\text { GIST avançado } \\
(n=9)\end{array}$} \\
\hline & $\mathbf{N}$ & $\%$ & $\mathbf{N}$ & $\%$ & $\mathbf{N}$ & $\%$ \\
\hline Idade (mediana) & \multicolumn{2}{|c|}{59} & \multicolumn{2}{|c|}{58} & \multicolumn{2}{|c|}{59} \\
\hline Intervalo (anos) & \multicolumn{2}{|c|}{$29-79$} & \multicolumn{2}{|c|}{$29-77$} & \multicolumn{2}{|c|}{$36-70$} \\
\hline$<=60$ & 16 & 50 & 14 & 60,8 & 2 & 22,3 \\
\hline$>60$ & 16 & 50 & 9 & 39,2 & 7 & 77,7 \\
\hline \multicolumn{7}{|l|}{ Sexo } \\
\hline Feminino & 16 & 50 & 12 & 52,1 & 4 & 44,5 \\
\hline Masculino & 16 & 50 & 11 & 47,9 & 5 & 55,5 \\
\hline \multicolumn{7}{|l|}{ Localização } \\
\hline Estômago & 15 & 46,9 & 12 & 52,1 & 3 & 33,3 \\
\hline Intestino delgado & 13 & 40,6 & 9 & 39,1 & 4 & 44,5 \\
\hline Esôfago & 1 & 3,2 & 1 & 4,4 & 0 & 0 \\
\hline Reto & 2 & 6,3 & 1 & 4,4 & 1 & 11,1 \\
\hline Desconhecido & 1 & 3,2 & 0 & 0 & 1 & 11,1 \\
\hline Tamanho (mediana) & \multicolumn{2}{|c|}{8,4} & \multicolumn{2}{|c|}{8} & \multicolumn{2}{|c|}{9,8} \\
\hline Intervalo (cm) & \multicolumn{2}{|c|}{$2,0-19$} & \multicolumn{2}{|c|}{$2,0-15$} & \multicolumn{2}{|c|}{$2,5-19$} \\
\hline$<5$ & 8 & 25 & 7 & 30,4 & 1 & 11,2 \\
\hline 5 a 10 & 13 & 40,6 & 9 & 39,1 & 4 & 44,4 \\
\hline$>10$ & 8 & 25 & 6 & 26 & 2 & 22,2 \\
\hline Desconhecido & 3 & 9,4 & 1 & 4,5 & 2 & 22,2 \\
\hline \multicolumn{7}{|c|}{ Índice mitótico (50 CGA) } \\
\hline$<5$ & 16 & 50 & 13 & 56,6 & 3 & 33,3 \\
\hline$>5$ & 9 & 28,1 & 5 & 21,7 & 4 & 44,4 \\
\hline Desconhecido & 7 & 21,9 & 5 & 21,7 & 2 & 22,3 \\
\hline \multicolumn{7}{|l|}{ Risco de agressividade } \\
\hline Baixo risco & 5 & 15,6 & 4 & 17,4 & 1 & 11,1 \\
\hline Risco intermediário & 6 & 18,7 & 5 & 21,7 & 1 & 11,1 \\
\hline Alto risco & 12 & 37,5 & 11 & 47,8 & 1 & 11,1 \\
\hline Metástase & 6 & 18,7 & 0 & 0 & 6 & 66,7 \\
\hline Desconhecido & 3 & 9,4 & 3 & 13,1 & 0 & 0 \\
\hline \multicolumn{7}{|l|}{ Imuno-histoquímica } \\
\hline CD117 positivo & 32 & 100 & 23 & 100 & 9 & 100 \\
\hline CD34 positivo & 26 & 81,2 & 19 & 82,6 & 7 & 77,8 \\
\hline \multicolumn{7}{|l|}{ Margem de ressecção } \\
\hline RO & 19 & 59,3 & 17 & 73,9 & 2 & 22,2 \\
\hline R1 & 8 & 25 & 5 & 21,7 & 3 & 33,3 \\
\hline Não avaliado & 5 & 15,7 & 1 & 4,4 & 4 & 44,5 \\
\hline \multicolumn{7}{|l|}{ Uso do imatinibe } \\
\hline Sim & 16 & 50 & 9 & 39,1 & 7 & 77,7 \\
\hline Não & 16 & 50 & 14 & 60,9 & 2 & 23,3 \\
\hline
\end{tabular}

Nota: GIST localizado - diagnóstico inicial de doença localizada; GIST avançado - diagnóstico inicial de doença metastática/risco de agressividade, Fletcher et al. ${ }^{3}$, categorizaçăo de risco do NIH / R0 - ausência de doença residual; R1- margem positiva ou doença residual/ 50CGA - por 50 campos de grande aumento 
A

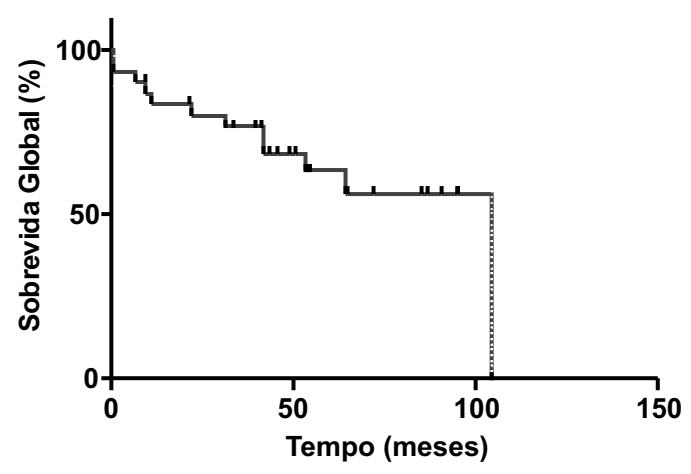

B

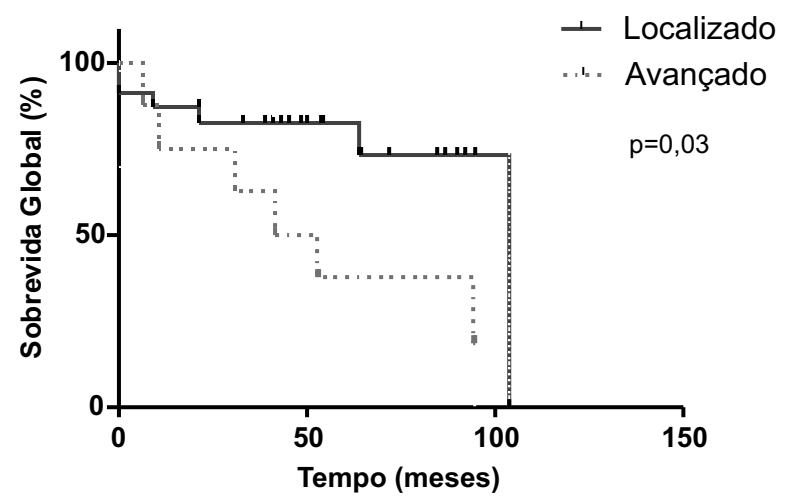

Figura 1. A- Sobrevida global do grupo total; B- Sobrevida global da doença localizada e doença avançada

Tabela 2. Características do Uso do Imatinibe

\begin{tabular}{l|c|c}
\multicolumn{1}{c|}{ Uso do Imatinibe } & N=16 & $\%$ \\
\hline Indicação & 1 & 6,2 \\
\hline Margem positiva & 2 & 12,5 \\
\hline Recorrência local & 6 & 37,5 \\
\hline Recorrência a distância & 7 & 43,8 \\
\hline $\begin{array}{l}\text { Metástase a distância no } \\
\text { diagnóstico }\end{array}$ & 0 & 0 \\
\hline Resposta & 6 & 37,6 \\
\hline Resposta completa & 8 & 50 \\
\hline Resposta parcial & 1 & 6,2 \\
\hline Doença estável & 1 & 6,2 \\
\hline Ausência de resposta & \multicolumn{2}{|c}{} \\
\hline Não avaliado & 7 & 43,7 \\
\hline Variação de Dose & 6 & 37,6 \\
\hline Manutenção da dose & 3 & 18,7 \\
\hline Aumento da dose (progressão) & \multicolumn{2}{|c}{} \\
\hline Redução da dose (toxicidade) & & \\
\hline & & \\
\hline
\end{tabular}

inicial (43,8\%); oito pacientes por recorrência; destes, seis a distância $(37,5 \%)$ e dois local $(12,5 \%)$; e um paciente por margem comprometida $(6,2 \%)$ (Tabela 3$)$. A dose inicial utilizada foi de $400 \mathrm{mg} / \mathrm{dia}$. O início do uso do imatinibe foi em setembro de 2003 . O benefício clínico ou controle da doença ocorreu em $87,6 \%$ dos pacientes, com $50 \%$ apresentando doença estável e 37,6\% resposta parcial. Ausência de resposta em um paciente $(6,2 \%)$ e nenhum com resposta radiológica completa.

Do total de 16 pacientes, nove foram a óbito $(56,2 \%)$ no ponto utilizado como censo e em um a causa não foi relacionada à doença (septicemia). A dose de $400 \mathrm{mg} /$ dia foi utilizada até a progressão e esta ocorreu em oito pacientes; destes, seis aumentaram a dose do imatinibe, três para $600 \mathrm{mg} /$ dia; e três para $800 \mathrm{mg} / \mathrm{dia}$; e na evolução todos apresentaram uma segunda progressáo. Desses pacientes, dois apresentaram óbito precoce (com 3 e 6 meses), dois aumentaram a dose de 600 para $800 \mathrm{mg} /$ dia, um com duraçáo de resposta de 12 meses e óbito, e o outro com duração de 46 meses, troca para sunitinibe e óbito) e os outros dois pacientes aumentaram de 400 para $800 \mathrm{mg} / \mathrm{dia}$ (um com resposta de 14 meses e após óbito, e o outro com resposta por 4,6 meses, troca para sunitinibe e encontrando-se vivo).

A sobrevida global em 3 anos foi de $81,2 \%$ e a sobrevida global em 5 anos de $51,5 \%$, com mediana de 53 meses (Figura 2). A sobrevida livre de primeira progressão foi de $55 \%$ em 3 anos e de $34,3 \%$ em 5 anos, com mediana de 32,9 meses e a sobrevida livre mediana de segunda progressão foi de 13 meses (Figura 2). Em uma análise comparativa entre os pacientes com metástase hepática $\mathrm{e}$ aqueles com recaída local e em peritônio isolado, foram encontradas diferença estatística na sobrevida livre de progressão $(\mathrm{p}=0,01)$ e tendência na sobrevida global a favor do grupo com recaída local e em peritônio isolada $(\mathrm{p}=0,06)$ (Figura2).

Quanto à tolerabilidade, ocorreu necessidade de redução da dose por toxicidade em três pacientes (18,7\%). Os efeitos colaterais mais frequentes relatados foram edema em oito pacientes (50\%), câimbras em cinco pacientes $(31,6 \%)$, dor epigástrica em três pacientes $(18,7 \%)$ e náuseas e tonturas em dois pacientes (12,5\%). Os efeitos colaterais, em sua maioria, foram considerados grau 1 pelos critérios de toxicidade pelo National Cancer Institute (NCI), com apenas dois pacientes com grau 3, um com anemia e outro com vômitos.

\section{DISCUSSÃO}

O GIST é uma neoplasia rara e devido ao sucesso do seu tratamento com a terapia molecular tornou-se um 
Tabela 3. Relação dos fatores prognósticos e sobrevida livre de doença e sobrevida global no GIST localizado

\begin{tabular}{|c|c|c|}
\hline GIST Localizado & Sobrevida Livre de Doença & Sobrevida Global \\
\hline Fatores Prognósticos (Comparações) & Valor do Log-rank P & Valor do Log-rank $P$ \\
\hline \multicolumn{3}{|l|}{ Idade } \\
\hline \multicolumn{3}{|l|}{$<60$ anos } \\
\hline$>60$ anos & 0,53 & 0,14 \\
\hline \multicolumn{3}{|l|}{ Sexo } \\
\hline \multicolumn{3}{|l|}{ Masculino } \\
\hline Feminino & 0,58 & 0,64 \\
\hline \multicolumn{3}{|l|}{ Tamanho } \\
\hline \multicolumn{3}{|l|}{$<5 \mathrm{~cm}$} \\
\hline$\geq 5 \mathrm{~cm}$ & 0,18 & 0,10 \\
\hline \multicolumn{3}{|l|}{ Localização } \\
\hline \multicolumn{3}{|l|}{ Estômago/Esôfago } \\
\hline Intestino Delgado/Reto & 0,96 & 0,23 \\
\hline \multicolumn{3}{|l|}{ Grau de Mitose } \\
\hline \multicolumn{3}{|l|}{$<5$ mitoses /CGA } \\
\hline$>5$ mitoses/CGA & 0,09 & 0,4 \\
\hline \multicolumn{3}{|l|}{ Risco de Agressividade } \\
\hline \multicolumn{3}{|l|}{ Baixo/Intermediário Risco } \\
\hline Alto Risco & 0,53 & 0,25 \\
\hline \multicolumn{3}{|l|}{ Doença residual } \\
\hline \multicolumn{3}{|l|}{ RO } \\
\hline R1 & 0,46 & 0,30 \\
\hline \multicolumn{3}{|l|}{ CD34 } \\
\hline \multicolumn{3}{|l|}{ Positivo } \\
\hline Negativo & 0,51 & 0,52 \\
\hline \multicolumn{3}{|l|}{ Recaída } \\
\hline \multicolumn{3}{|l|}{ Sim } \\
\hline Não & - & 0,20 \\
\hline
\end{tabular}

tumor de imenso interesse para a realização desta pesquisa. Foram 32 pacientes estudados com diagnóstico em um período de 5 anos e com um seguimento mediano de 43,7 meses. Esse número de pacientes foi considerado expressivo devido tratar-se de um centro único. As maiores séries brasileiras encontradas publicadas com avaliação da evoluçâo clínica foram do Instituto Nacional de Câncer com 94 pacientes em um período de 14 anos e do Hospital de Clínicas de Porto Alegre com 85 pacientes em um período de 17 anos, uma outra série com 513 pacientes de um centro laboratorial de diagnóstico nacional avaliou principalmente dados anatomopatológicos ${ }^{13-15}$.

A idade mediana ao diagnóstico de 59 anos e a não ocorrência de diferença na incidência entre os sexos foram comparáveis aos dados da literatura ${ }^{2,16}$. A maioria dos pacientes apresentava sintomas como dor abdominal e sangramento gastrointestinal, sendo apenas $9,3 \%$ assintomáticos. Esse dado pode ser explicado pelo tamanho volumoso mediano de $8,4 \mathrm{~cm}$ das lesóes, em um estudo sueco com 288 pacientes, $20 \%$ eram assintomáticos e $10 \%$ achados de necropsia ${ }^{4}$. Na doença localizada, o sítio mais frequente foi estômago em $54,1 \%$ e na doença avançada inicial o sítio mais frequente foi o intestino delgado em $44,5 \%$. Esse dado pode estar relacionado com o pior prognóstico da localizaçáo no intestino delgado, essa característica conhecida por estudos anteriores fez com que o sítio anatômico fosse incorporado em uma outra classificação ao risco de comportamento agressivo do GIST ${ }^{16}$.

O tamanho tumoral e o índice mitótico são os dois principais fatores na estratificaçáo de risco de comportamento agressivo do GIST ${ }^{3}$. O índice mitótico 


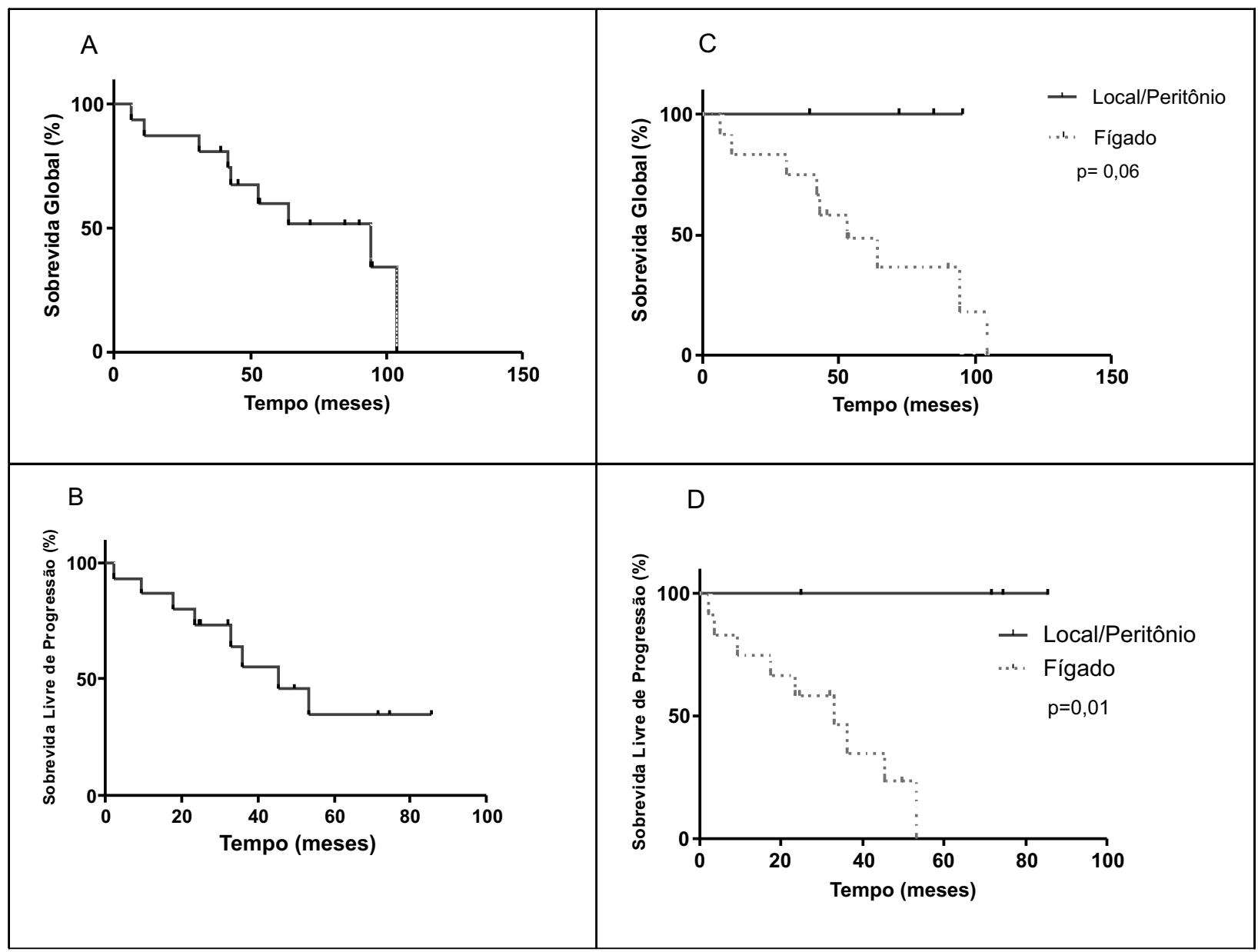

Figura 2. A- Sobrevida global do grupo que utilizou imatinibe; B- Sobrevida livre de progressão do grupo que utilizou imatinibe; C e DDiferença de sobrevida global e livre de doença entre pacientes com metástase em fígado versus metástase peritonial e recaída local e uso do imatinibe

foi desconhecido em $21,9 \%$, esse número é considerado alto, tratando-se de uma análise considerada simples pelo patologista e de extrema importância para a avaliaçáo prognóstica dessa neoplasia, este fato mostra desconhecimento e também foi observado em um outro estudo brasileiro ${ }^{17}$. Quanto à classificação nas categorias de risco, apresentou-se uma maior incidência de tumores de alto risco, $37,5 \%$, dado náo observado em outros estudos publicados ${ }^{4,18}$. Entretanto, em revisão de seis estudos com mais de 100 pacientes, foi observada uma média de alto risco de $44 \%$, comparável ao presente estudo ${ }^{19}$.

Para melhor análise e também por se tratar de dois grupos totalmente diferentes, os pacientes foram separados em doença localizada e doença avançada no diagnóstico. A ressecção cirúrgica completa consiste no tratamento principal do GIST localizado; a ressecção R0, com ausência de doença residual, é essencial para o bom resultado do tratamento ${ }^{8}$. No presente estudo, dos 23 pacientes com doença localizada, $18,1 \%$ apresentavam margens comprometidas e, destes, $75 \%$ apresentaram recidiva; na literatura, a taxa de $\mathrm{R} 0$ apresenta grande variação, de $9,3 \%$ até $53 \%$ 2,20. A taxa de recaída do grupo de GIST localizado foi de 39,1\%, essa taxa de recaída foi semelhante a de alguns estudos que variou de $40 \%$ a mais de $50 \%^{2,8}$. Devido a essa alta taxa de recaída, existe a proposta do tratamento adjuvante com o imatinibe; no presente estudo, apenas um paciente recebeu adjuvância por ter apresentado margens comprometidas. O estudo ACOSOG Z9001 com 713 pacientes randomizou os pacientes com GIST localizado maior do que $3 \mathrm{~cm}$ para imatinibe $400 \mathrm{mg} / \mathrm{dia}$ ou placebo e mostrou uma sobrevida livre de recaída de $98 \%$ versus $83 \%(\mathrm{p}<0,0001)$ em 1 ano, sem diferença na sobrevida global, sua atualizaçáo em 2010 mostrou benefício no risco intermediário e alto risco ${ }^{21}$.

$\mathrm{Na}$ doença localizada inicial, a sobrevida global em 5 anos foi de $73,2 \%$ e a sobrevida livre de doença de $73,8 \%$, essas sobrevidas são semelhantes a alguns estudos com doença localizada ${ }^{18,22}$. Esse número foi considerado satisfatório, provavelmente devido à alta taxa de uso do imatinibe na recaída; dos oito pacientes, $100 \%$ utilizaram. $\mathrm{Na}$ doença avançada, a sobrevida global em 5 anos foi de $37,5 \%$, sendo que $77,7 \%$ utilizaram o imatinibe, esse grupo também não é semelhante a outros da literatura 
para comparação; pois, os maiores estudos são de uso de imatinibe, na recaída e doença metastática inicial, que será o último tema desta discussão.

Os fatores prognósticos no GIST são um tópico extensamente estudados, com alguns fatores bem estabelecidos como de pior prognóstico como sexo masculino, tamanho tumoral elevado, alto índice mitótico, localização em intestino delgado e mutação do éxon $9^{2-4,6,16}$. No presente estudo, foi realizada análise estatística dos subgrupos e sua influência na sobrevida e, em nenhum, foi encontrado significância estatística, isto ocorreu provavelmente devido ao pequeno número da amostra, consistindo em uma limitação do estudo.

A experiência do uso do imatinibe no GIST avançado não foi publicada por nenhum centro brasileiro, em pesquisa no Lilacs. Este estudo, apesar de se tratar apenas de 16 pacientes em uso de imatinibe, consiste de dados importantes, o seu uso foi iniciado em 2003, 1 ano após o primeiro estudo fase II ser publicado; o seguimento mediano foi longo, de 43,7 meses; nenhum paciente deste grupo perdeu o seguimento e alguns pacientes chegaram até a terceira linha de tratamento. A dose inicial utilizada foi de $400 \mathrm{mg} / \mathrm{dia}$, dose atualmente confirmada como suficiente com os dados recentes do estudo MetaGIST, que não mostrou diferença na sobrevida global comparando-se com a dose de $800 \mathrm{mg} / \mathrm{dia}^{23}$. O controle da doença ocorreu em $87,6 \%$, com doença estável em $50 \%$ e resposta parcial em $37,6 \%$, semelhante a outros estudos que variaram de $74 \%$ a $90 \%{ }^{1}$.

A sobrevida global em 5 anos foi de $51,5 \%$, com mediana de 53 meses, com resultado semelhante a dois grandes estudos multicêntricos americanos e europeus com 746 e 147 pacientes, que mostraram sobrevidas medianas de 55 e 57 meses $^{7,12}$. A sobrevida livre de progressão mediana foi de 32,9 meses, maior que nos estudos citados anteriormente que foram de 18 e 20 meses, essa diferença pode ter sido pela melhor detecçáo da progressão, não apenas pelo critério RECIST como neste estudo, mas pelos critérios de Choi náo utilizados no presente estudo ${ }^{7,11-12}$. Os pacientes utilizaram o imatinibe $400 \mathrm{mg} /$ dia até a progressão, dado validado pelo estudo recente francês que comparou o uso contínuo versus a parada em 3 anos e encontrou um alto risco de progressão rápida após a parada ${ }^{22}$.

A progressão após imatinibe ocorreu em $50 \%$ dos pacientes e, destes, $75 \%$ aumentaram a dose, na metade para $600 \mathrm{mg} /$ dia, e o restante para $800 \mathrm{mg} / \mathrm{dia}$, observou-se resposta nos dois grupos, não foi possível avaliar estatisticamente a diferença na duraçáo de resposta entre as doses devido ao pequeno número de pacientes. O estudo fase III S0033 planejou o escalonamento de dose para $800 \mathrm{mg} /$ dia nos pacientes que progrediram com $400 \mathrm{mg} / \mathrm{dia}$ e um terço dos pacientes obteve algum benefício, mas não existe um estudo que comparou a dose de 600 com 800 $\mathrm{mg} / \mathrm{dia}^{7}$. O papel do sunitinibe na doença resistente ao imatinibe foi demonstrado em um estudo randomizado de fase III que comparou sunitinibe versus placebo em pacientes refratários a imatinibe e mostrou aumento na sobrevida global, com redução do risco de morte de aproximadamente $50 \%$, mas a resposta ao sunitinibe no tratamento de resgate foi pouco duradoura ${ }^{24}$. No presente estudo, dois pacientes utilizaram o sunitibe, um paciente por dois meses e o outro encontrava-se em uso por três meses. O imatinibe foi bem tolerado, apresentando na maioria toxicidade grau 1 , o efeito mais comum observado foi edema e a redução de dose ocorreu em 18,7\% dos pacientes, dados similares à literatura, e nenhum efeito adverso sério foi observado ${ }^{7,12,25}$.

\section{CONCLUSÃO}

O presente estudo mostrou que a maioria dos tumores foi grande, com mediana de $8,4 \mathrm{~cm}$, de localização gástrica e de alto risco de agressividade. A taxa de recaída na doença localizada foi alta, de 40,9\%, mas semelhante a outros centros internacionais e relacionada à própria biologia $\mathrm{da}$ doença e em parte podendo ser diminuída provavelmente com o acréscimo da adjuvância com o imatinibe. Nos pacientes com doença localizada, não ocorreu significância estatística entre os fatores prognósticos e a sobrevida, provavelmente relacionada ao tamanho da amostra. $\mathrm{O}$ tratamento com o imatinibe mostrou resultados excelentes, comparáveis aos maiores centros americanos, e uma baixa perda de seguimento, uma preocupaçáo constante de países em desenvolvimento. Apesar desses resultados, ainda ocorre uma alta recaída na doença localizada e a doença avançada na maioria dos pacientes náo é curável, devido à ocorrência de refratariedade ao imatinibe. Assim, deve ocorrer a continuidade das diversas pesquisas procurando ultrapassar essas barreiras ainda existentes.

\section{CONTRIBUIÇÕES}

Karina Costa Maia Vianna contribuiu na concepção e no planejamento do estudo; na obtenção, análise e interpretação dos dados; assim como na redação final da versão publicada; Sérgio Lunardon Padilha contribuiu na análise e interpretação dos dados; na aplicação estatística, assim como na revisão crítica e aprovação final da versão publicada; Bianca Ramos Ferronatto, Lucas Fernando Uratani, Felipe Augusto Santiago de Almeida contribuíram na obtenção dos dados em prontuário; José Zanis Neto contribuiu na concepção e no planejamento do estudo; na análise e interpretação dos dados; assim como na redação e revisão crítica e aprovação final da versão publicada.

Declaraçáo de Conflito de Interesses: Nada a Declarar. 


\section{REFERÊNCIAS}

1. Rubin BP, Heinrich MC, Corless CL. Gastrointestinal stromal tumour. Lancet. 2007;369(9574):1731-41.

2. DeMatteo RP, Lewis JJ, Leung D, Mudan SS, Woodruff JM, Brennan MF. Two hundred gastrointestinal stromal tumors: recurrence patterns and prognostic factors for survival. Ann Surg. 2000;231(1):51-8.

3. Fletcher CD, Berman JJ, Corless C, Gorstein F, Lasota J, Longley BJ, et al. Diagnosis of gastrointestinal stromal tumors: a consensus approach. Int J Surg Pathol. 2002; 10(2):81-9.

4. Nilsson B, Bümming P, Meis-Kindblom JM, Odén A, Dortok A, Gustavsson B, et al. Gastrointestinal stromal tumors: the incidence, prevalence, clinical course, and prognostication in the preimatinib mesylate era--a population-based study in western Sweden. Cancer. 2005;103(4):821-9.

5. Tryggvason G, Gíslason HG, Magnússon MK, Jónasson JG. Gastrointestinal stromal tumors in Iceland, 19902003: the icelandic GIST study, a population-based incidence and pathologic risk stratification study. Int J Cancer. 2005;117(2):289-93.

6. Fletcher CD, et al. Prognostic value of KIT mutation type, mitotic activity, and histologic subtype in gastrointestinal stromal tumors. J Clin Oncol. 2002;20(18):3898-905.

7. Blanke CD, Rankin C, Demetri GD, Ryan CW, Mehren $\mathrm{M}$, Benjamin RS, et al. Phase III randomized, intergroup trial assessing imatinib mesylate at two dose levels in patients with unresectable or metastatic gastrointestinal stromal tumors expressing the kit receptor tyrosine kinase: S0033. J Clin Oncol. 2008;26(4):626-32.

8. Valadão M, Linhares E, Mali Jr. J, Sousa J, Stoduto G. Novas perspectivas no tratamento do GIST. Rev bras cancerol. 2006;52(4):373-9.

9. Mudan SS, Conlon KC, Woodruff JM, Lewis JJ, Brennan MF. Salvage surgery for patients with recurrent gastrointestinal sarcoma: prognostic factors to guide patient selection. Cancer. 2000;88(1):66-74.

10. Joensuu H, Roberts PJ, Sarlomo-Rikala M, Andersson LC, Tervahartiala P, Tuveson D, et al. Effect of the tyrosine kinase inhibitor STI571 in a patient with a metastatic gastrointestinal stromal tumor. N Engl J Med. 2001;344(14):1052-6.

11. Le Cesne A, Van Glabbeke M, Verweij J, Casali PG, Findlay $\mathrm{M}$, Reichardt $\mathrm{P}$, et al. Absence of progression as assessed by response evaluation criteria in solid tumors predicts survival in advanced GI stromal tumors treated with imatinib mesylate: the intergroup EORTC-ISG-AGITG phase III trial. J Clin Oncol. 2009;27(24):3969-74.

12. Blanke CD, Demetri GD, Mehren M, Heinrich MC, Eisenberg B, Fletcher JA, et al. Long-term results from a randomized phase II trial of standard- versus higherdose imatinib mesylate for patients with unresectable or metastatic gastrointestinal stromal tumors expressing KIT. J Clin Oncol. 2008;26(4):620-5.

13. Guimarães DP, Braggio E, Bacchi CE, Lopes LF, Small IA, Valadão CM, et al. Clinical and morphological characterization of GIST patients (pts) in Brazil. J Clin Oncol. 2004;22(14 Suppl):4178.

14. Cerski MR. Tumores estromais gastrointestinais (GIST): fatores de risco e análise molecular de 85 casos provenientes do Hospital de Clínicas de Porto Alegre, Rio Grande do Sul [tese]. Porto Alegre: Universidade Federal do Rio Grande do Sul, Faculdade de Medicina, Programa de Pós-Graduação em Medicina: Ciências em Gastroenterologia; 2010.

15. Lopes LF, Ojopi EB, Bacchi CE. Gastrointestinal stromal tumor in Brazil: clinicopathology, immunohistochemistry, and molecular genetics of 513 cases. Pathol Int. 2008;58(6):344-52.

16. Miettinen M, Lasota J. Gastrointestinal stromal tumors: pathology and prognosis at different sites. Semin Diagn Pathol. 2006;23(2):70-83.

17. Valadão M, Linhares E, Monteiro M, Fernandes DS, Vidal EI, Saraiva CF, et al. Perfil dos portadores de GIST operados no Estado do Rio de Janeiro: estudo multicêntrico SBCO. Rev bras cancerol. 2009;55(2):145-9

18. Cho MY, Sohn JH, Kim JM, Kim KM, Park YS, Kim $\mathrm{WH}$, et al. Current trends in the epidemiological and pathological characteristics of gastrointestinal stromal tumors in Korea, 2003-2004. J Korean Med Sci. 2010;25(6):853-62.

19. Joensuu H. Risk stratification of patients diagnosed with gastrointestinal stromal tumor. Hum Pathol. 2008;39(10):1411-9.

20. Miettinen M, Sobin LH, Lasota J. Gastrointestinal stromal tumors of the stomach: a clinicopathologic, immunohistochemical, and molecular genetic study of 1765 cases with long-term follow-up. Am J Surg Pathol. 2005;29(1):52-68.

21. DeMatteo RP, Ballman KV, Antonescu CR, Maki RG, Pisters PWT, Demetri GD, et al. Placebo-controlled randomized trial of adjuvant imatinib mesylate following the resection of localized, primary gastrointestinal stromal tumor (GIST). Lancet. 2009; 373(9669):1097-104.

22. Le Cesne A, Ray-Coquard I, Bui BN, Adenis A, Rios M, Bertucci $F$, et al. Discontinuation of imatinib in patients with advanced gastrointestinal stromal tumours after 3 years of treatment: an open-label multicentre randomised phase 3 trial. Lancet Oncol. 2010;11(10):942-9.

23. Van Glabbeke M, Verweij J, Blay JY, Demetri G, Heinrich M, Borden E, et al. Comparison of two doses of imatinib for the treatment of unresectable or metastatic gastrointestinal stromal tumors: a meta-analysis of 1,640 patients. J Clin Oncol. 2010;28(7):1247-53.

24. Demetri GD, Oosterom AT, Garrett CR, Blackstein ME, Shah $\mathrm{MH}$, Verweij J, et al. Efficacy and safety of sunitinib in patients with advanced gastrointestinal stromal tumour after failure of imatinib: a randomised controlled trial. Lancet. 2006;368(9544):1329-38.

25. Demetri GD, Mehren M, Blanke CD, Van den Abbeele AD, Eisenberg B, Roberts PJ, et al. Efficacy and safety of imatinib mesylate in advanced gastrointestinal stromal tumors. N Engl J Med. 2002;347(7):472-80. 


\begin{abstract}
Introduction: Gastrointestinal stromal tumors (GIST) are rare neoplasms that originate from interstitial cells of Cajal. Objectives: To describe the experience at the Curitiba Clinical Hospital in the treatment of both localized and advanced GIST, with analysis of clinical and anatomical and pathological characteristics and use of imatinib. Methodology: A retrospective study of 32 patients diagnosed for immunohistochemistry, c-Kit positive, from 2003 to 2008. Results: The median age was 66 years, the median tumor size was $8.4 \mathrm{~cm}$ and the most common sites were the stomach in $46.9 \%$ and the small intestine in $40.9 \%$. Patients at high risk of progressive disease: $37.5 \% .23$ patients had localized disease at diagnosis, 39.1\% relapsed, and 9 patients had advanced disease. The median follow up was 43.7 months, overall survival at 5 years for the total group of $56.2 \%$. In localized disease, overall survival at 5 years was $73.8 \%$ and $37.5 \%$ in advanced disease $(\mathrm{p}=0.03)$. There was no impact of prognostic factors on survival. The use of imatinib occurred in 16 patients, $43.8 \%$ due to initial metastasis, $37.5 \%$ disease progression, $12.5 \%$ local recurrence and $6.2 \%$ compromised margins. The median overall survival with the use of imatinib was 53 months and the first progression-free survival of 32.9 months. There was good tolerance to imatinib and only two patients received sunitinib. Conclusion: Predominant tumors were large, gastric-sited and of high risk of progression. The relapse rate was high in localized disease. And overall survival of patients with localized disease and who used the imatinib was considered satisfactory. Key words: Gastrointestinal Stromal Tumors; Antineoplastic Agents/therapeutic use; Mesylates; Proto-Oncogene Proteins c-kit; Immunohistochemistry; Retrospective Studies
\end{abstract}

\title{
Resumen
}

Introducción: Los tumores del estroma gastrointestinales (GIST) son neoplasias raras que se originan en las células intersticiales de Cajal. Objetivo: Describir la experiencia del Hospital de Clínicas de Curitiba en el tratamiento de tumores de GIST localizado y avanzado, con análisis de las características clínicas, anatómicas y patológicas, además del uso de imatinib. Método: Estudio retrospectivo de 32 pacientes con diagnóstico por inmune histoquímica, c-Kit positivo, en el período de 2003 a 2008. Resultados: Franja etaria de 66 años, el tamaño mediano del tumor $8,4 \mathrm{~cm}$ y los locales más frecuentes fueron el estómago en $46,9 \%$ e intestino delgado en $40,9 \%$. Pacientes con alto riesgo de agresividad: el 37,5\% presentaban enfermedad localizada en el diagnóstico de 23 pacientes: $39,1 \%$ recayeron y 9 de enfermedad avanzada. El resultado mediano fue de 43,7 meses, con supervivencia global en 5 ańos en el grupo total de 56,2\%. En cuanto a la enfermedad localizada, la supervivencia en 5 años fue de $73,8 \%$ y en la avanzada de $37,5 \%(\mathrm{p}=0,03)$. No hubo un impacto de los factores pronósticos en la supervivencia. El mesilato de imatinib fue usado en 16 pacientes: el 43,8\% por metástasis inicial, el 37,5\% recaída a distancia, el 12,5\% recaída local y el 6,2\% margen afectada. La supervivencia global con el uso del imatinib mediana fue de 53 meses y la supervivencia libre de primera progresión de 32,9 meses. Hubo buena tolerabilidad al imatinib y solo dos pacientes utilizaron el sunitinib. Conclusión: La mayoría de los tumores era grande, de localización gástrica y de alto riesgo de agresión. La tasa de recaída es alta en la enfermedad localizada. Y la supervivencia global de pacientes con enfermedad localizada y que utilizaron el imatinib se considera satisfactoria.

Palabras clave: Tumores del Estroma Gastrointestinal; Agentes Antineoplásicos/uso terapéutico; Mesilatos; Proteínas Proto-Oncogénicas c-kit; Inmunohistoquímica; Estudios Retrospectivos 